\title{
Personality pathology recorded by severity: national survey
}

\author{
Min Yang, Jeremy Coid and Peter Tyrer
}

\section{Background}

Current classifications of personality disorders do not classify severity despite clinical practice favouring such descriptions.

\begin{abstract}
Aims
To assess whether an existing measure of severity of personality disorder predicted clinical pathology and societal dysfunction in a community sample.
\end{abstract}

\section{Method}

UK national epidemiological study in which personality status was measured using the screening version of the Structured Clinical Interview for DSM-IV Personality Disorders (SCID-II) and reclassified to five levels using a modified severity index. Associations between levels of severity of personality pathology and social, demographic and clinical variables were measured.

\section{Results}

Of 8391 individuals interviewed and their personality status assessed, only a minority $(n=1933,23 \%)$ had no personality pathology. The results supported the hypothesis. More severe personality pathology was associated incrementally with younger age, childhood institutional care, expulsion from school, contacts with the criminal justice system, economic inactivity, more Axis I pathology and greater service contact (primary care and secondary care, all $P<0.001$ ). Significant handicap was noted among people with even low levels of personality pathology. No differences contradicted the main hypothesis.

\section{Conclusions}

A simple reconstruction of the existing classification of personality disorder is a good predictor of social dysfunction and supports the development of severity measures as a critical requirement in both DSM-V and ICD-11 classifications.

\section{Declaration of interest}

P.T. is the Chair of the World Psychiatric Association Section on Personality Disorders and the Chair of the World Health Organization Personality Disorder Working Group for the ICD-11 Classification. He is also Editor of the British Journal of Psychiatry but had no part in any decisions about this paper.
Epidemiological studies of personality disorder consistently demonstrate that between 4 and $12 \%$ of the adult population have a formal diagnosis of personality disorder. ${ }^{1-3}$ This level of prevalence of what is considered significant disorder is higher than clinical practice suggests and yet fails to identify much of personality pathology in the population. ${ }^{4}$ Interpretation is also made difficult by the absence of any measure of severity of personality disturbance, and as interventions for the condition are generally resource intensive there is currently no good way of selecting those who are in the most need. Studies among people with personality disorder in clinical practice have suggested that greater severity can be identified by the extent of comorbidity between categories of personality disorder ${ }^{5-7}$ with those with more named disorders, particularly those which cross the clusters A, B and C, having more complex disorders. Among this group, those with additional antisocial personality disorder have the most severe form of the condition. These studies have been relatively small in number but they have predicted both additional pathology and outcome successfully. It was therefore important to extend this enquiry to a larger epidemiological sample to determine whether similar associations with severity were found.

\section{Method}

The British National Survey of Psychiatric Morbidity assessed people aged 16-74 years living in private houses ${ }^{8}$ in which the sampling frame was the Royal Mail's small-users postcode address file for private households, with postcode sectors stratified within each National Health Service region on the basis of socioeconomic profile. Initially, 438 postal sectors were selected with a probability proportional to size, i.e. the number of delivery points. Postal sectors contain on average 2550 of these. Within each of these sectors, 36 were selected, yielding a sample of 15804 delivery points. These were visited to identify private households with at least one person aged 16-74 years. The Kish grid $\operatorname{method}^{9}$ was used to select systematically one person in each household. In phase I of the survey, participants completed computer-assisted interviews with Office for National Statistics interviewers in an interview lasting on average $1.5 \mathrm{~h}$. A total of 8886 adults completed a first-phase interview, a response rate of $69.5 \%$.

\section{Measures recorded}

Demographic, development and service use details

Questions were included in the screening phase I on self-reported healthcare service use, criminal justice involvement and placement in local authority and institutional care in childhood.

\section{Mental health status}

Possible psychotic disorder was also assessed in the first-phase interview using a combination of data from the Psychosis Screening Questionnaire, ${ }^{10}$ a history of taking antipsychotic medication or in-patient care. $^{3}$ Symptoms of other mental disorders were also assessed using the revised version of the Clinical Interview Schedule. ${ }^{11}$

\section{Personality status}

The screening questionnaire of the Structured Clinical Interview for DSM-IV Personality Disorders (SCID-II) ${ }^{12}$ was used to identify possible cases of personality disorder. Participants themselves entered 'yes' or 'no' responses to 116 questions on a laptop 
computer. This generated categories of DSM-IV ${ }^{13}$ personality disorder, and subsequent allocation to the three main clusters ${ }^{14}$ by applying algorithms developed using data obtained from the Structured Clinical Interview administered by trained interviewers in a previous survey of prisoners. ${ }^{15}$ The three clusters are not exclusive, and one person can have a diagnosis of personality disorders in more than one cluster. In the original study a second phase of interviews was then carried out in a weighted sample ${ }^{3}$ but in our study all the data from the screening phase were included. Severity of personality disturbance was determined by the Tyrer \& Johnson method ${ }^{5,6}$ in which five levels were identified; 0 (no personality disturbance), 1 (personality difficulty (one criterion less than the threshold for personality disorder)), 2 (simple personality disorder (in one DSM cluster only)), 3 (complex personality disorder (two or more personality disorders in more than one DSM cluster)) and 4 (severe personality disorder (two or more personality disorders in more than one DSM cluster with one being antisocial personality disorder)). The last group (level 4) puts antisocial personality disorder at the top of the hierarchy of personality disorders and makes an assumption that the most severe personality disorders create a risk to others.

Although the formal diagnoses of personality disorder were only made in the second phase of the study it was felt to be more appropriate to examine the full set of data from the first phase even though it was recognised that the prevalence figures would be overestimates as they were derived from a screening instrument alone. Previous data suggest that this inflates the diagnosis of personality disorder in clinical populations by around $20 \%$ compared with diagnoses developed from the full structured SCID-II interview. ${ }^{16}$

The main hypothesis tested was that those with more severe levels of personality disturbance would demonstrate greater disruption of social dysfunction and morbidity measured by criminal behaviour, employment history and use of health services, and show a greater association with mental state (Axis I) disorders.

\section{Statistical analysis}

The weighted prevalences of the severity of personality disturbance separated by gender, age and DSM-IV personality clusters were calculated using appropriate sampling weighting to reflect the population characteristics. Weighted prevalences of employment, deprivation factors or problem behaviours, common Axis I mental disorders and use of health services $\times$ severity level of personality disturbance were also calculated respectively for descriptive purposes. The detailed weighting procedures are published elsewhere. ${ }^{8}$ Based on weighted numbers, differences in the distribution of severity of personality $\times$ gender were further tested using Pearson's chi-squared test. The relationship between personality disturbance and age of participants was further tested using the chi-squared linearity test at each level of the severity scale. To test the possible graded relationship between the severity level and the level of economic activities by employment, of health service use, of Axis I mental disorders and deprivation factors, the same chi-squared linearity statistic was used. These simple and descriptive analyses were conducted in SPSS v12.0 for Windows.

However, as is often true of data from surveys, the association between personality disturbance and other relevant factors of interest (employment, health service use and deprivation factors) could be confounded by differences in the age and gender of participants. Similarly, the association between personality disturbance and Axis I mental disorders needs to take into account comorbidity of those disorders. Further analysis of the relationship between personality disturbance and these other factors was therefore felt to be necessary after adjusting for confounding and comorbidity.

Given five levels of severity and structured data by postal areas, multilevel multinomial logistic regression ${ }^{18}$ was used to allow for possible random effects of the postcode address file areas in the personality severity distribution and adjusting for confounding when such adjustments became appropriate. For example, for the association between employment and personality disturbance, we adjusted for age, gender and Axis I disorders. For the association between each Axis I disorder and personality disturbance, we adjusted for age, gender, employment and other Axis I disorders. By estimating odds ratios between one level of the severity and the reference level (typically the no personality disturbance category), the model enabled us to estimate the differential association between each of the factors of interest and each level of personality disturbance, and allowed graded effects of severity to be quantified and tested using the generalised Wald chi-squared test. The statistical package MLwiN (version 2.01 for Windows) was used for the multinomial regression analysis. $^{19}$

\section{Results}

\section{Overall prevalence and distribution of personality disturbance by age and gender}

Weighted numbers and prevalences are shown except where stated. Only $1933(23.0 \%)$ of the total sample (8391) screened were found to have no evidence of personality disturbance (i.e. level 0). Personality difficulty $(n=4055)$ was the most predominant group $(48.3 \%)$ and severe personality disorder $(n=110)$ the least $(1.3 \%)$. In clusters A and B more men than women had severe personality disorder but women had more complex personality disorders; there were no significant differences in cluster C personalities (Table 1), with older people having fewer complex and severe personality disorders but more personality difficulty (Table 2). The prevalence of clusters A and C showed little age change but that for cluster B personality disorder was reduced as age increased (Table 2), with much lower prevalence and reduced risk among those aged 35 and above (odds ratio $(\mathrm{OR}=0.31,95 \% \mathrm{CI} 0.25-0.37)$ compared with that of Cluster A $(\mathrm{OR}=1.00,95 \%$ CI $0.89-1.14)$ and of Cluster C $(\mathrm{OR}=1.06,95 \%$ CI $0.93-1.21)$.

\section{Relationship between deprivation, criminal and antisocial behaviour and severity of personality disturbance}

With a rate set at 1.0 for those with no personality disorder the equivalent rates for those with severe personality disorder were many times higher, with conduct disorder, criminal convictions, running away from home and homelessness showing the strongest relationship with severe disorder (Table 3). For all of these factors the level of personality disorder demonstrated a graded relationship with the abnormal behavioural features.

A two-level multinomial regression model was fitted for each of the deprivation factors on the risk at each level of the severity scale. The model took into account any possible random area effects and was adjusted for age, gender, Axis I mental disorders and substance misuse problems. The hypothesis that there would be less risk at low levels of personality disturbance and much greater risk at higher levels was supported strongly by the evidence. This was confirmed by the adjusted odds ratios as well as by the overall test statistic for the comparison of the odds ratios estimates between any two neighbouring levels on the dimensional scale (i.e. simple disorder $v$. personality difficulty, complex 

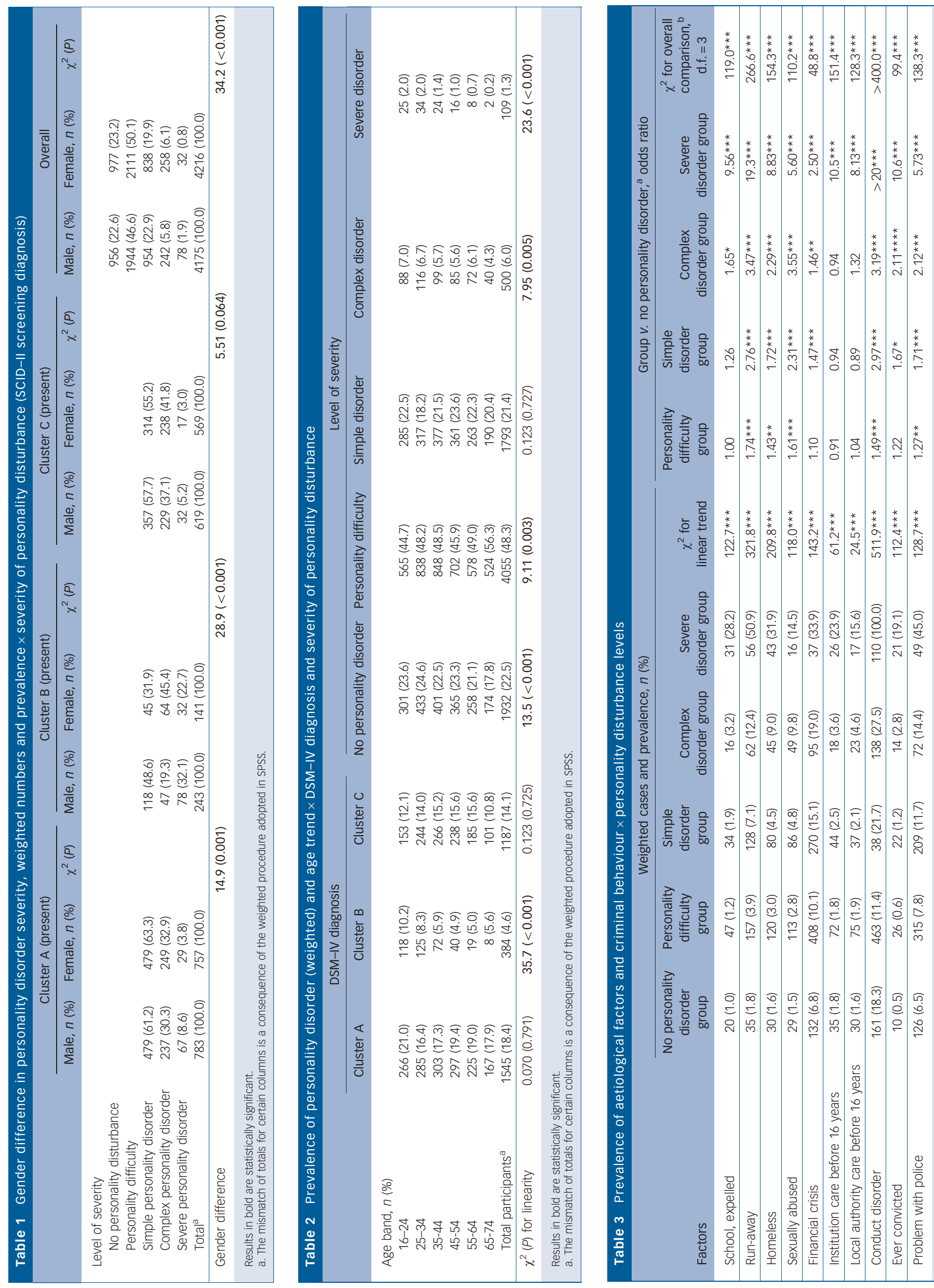

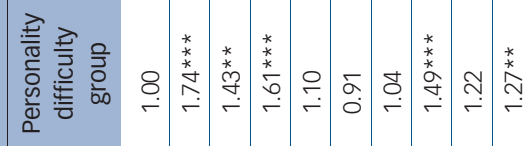

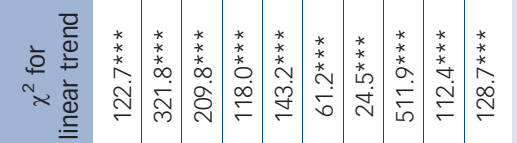

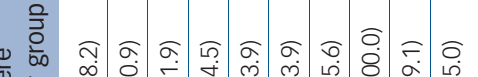

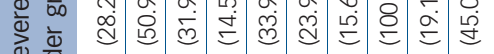

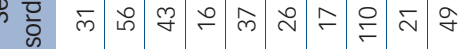

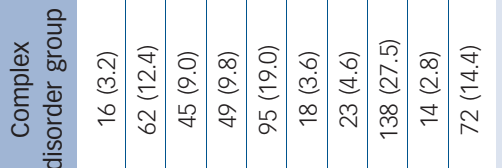

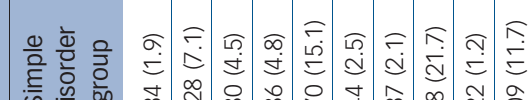

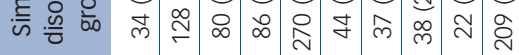

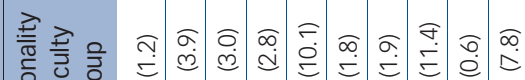

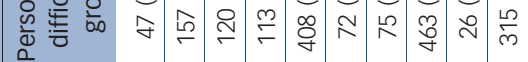

?

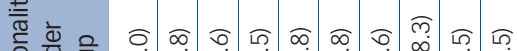

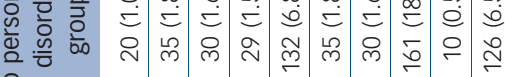
之

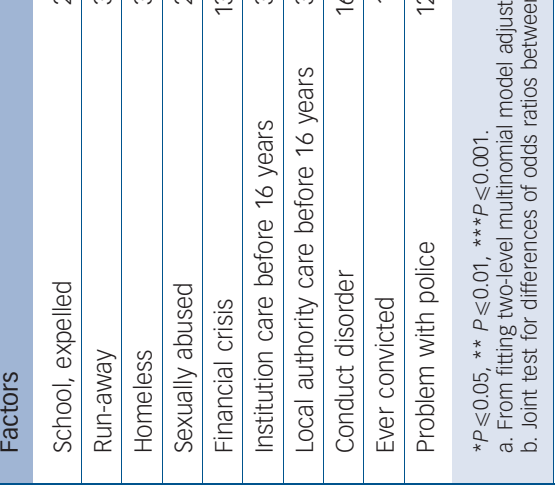


disorder $v$. simple disorder, severe disorder $v$. complex disorder). However, the incremental risk at the first four levels of the severity scale was considered moderate, with the most marked increment occurring between complex and severe personality disorders. Thus the risk of deprivation factors in association with severe personality disorder was between 1.7 times (financial crisis) and 11.2 times (institutional care) higher than that of complex personality disorder.

\section{Employment and personality disturbance}

There was a higher unemployment rate among participants with severe personality disorder than among those with no personality disorder or with simple disorders (Table 4). A two-level multinomial logistic model with the adjustments for age, gender, Axis I disorder and substance misuse estimated the impact of severity of personality disturbance on employment by odds ratios as demonstrated in Table 4. At the low level of the severity scale, there was no evidence of employment difference. As personality disturbance became more severe, the likelihood of being in parttime employment or unemployed, or with no economic activity, became greater when compared with full-time employment. This finding further confirmed the hypothesis of a graded relationship between severity of personality disturbance and economic activity.

\section{Axis I disorders, service use and personality disturbance}

Axis I pathology and use of all health services by participants was greater in those with more severe personality disturbance (Tables 5 and 6). However, for each service the sample size for severe personality disorder was small. For individuals with probable psychosis and the common mental (neurotic) disorders there was a doubling of the odds ratio for association at each level in the personality severity hierarchy and very high association of drug dependence with severe personality disorder.

Further multinomial regression analysis, with adjustments for Axis I conditions (Table 5), shows a moderate increment in service use with increasing severity (i.e. 'admitted to hospital for mental problems', 'seen social worker in the past year' and 'sought any professional help'), but the overlapping confidence intervals between the odds ratio for the last service item between complex and severe personality disorders suggested there were no significant differences in service use between the two levels of severity.

\section{Discussion}

The results of this survey suggest that personality disturbance measured by a broad screen is widespread and common, is associated with considerable psychiatric morbidity and social, occupational and personal handicaps, and is economically and usefully classified in terms of severity. This study has limitations. Although the findings suggest that the handicaps created by personality disorders are at least as great as those of Axis I disorders we have no means of knowing the direction of the association and whether some personality pathology is a direct reflection of mental state pathology. We also acknowledge that the SCID screen for personality disorder, like almost all screening instruments, overdiagnoses personality pathology but this only has the effect of altering the thresholds for the levels of pathology in our proposed system, not nullifying the differences between them. At first sight the finding that $72 \%$ of the population has at least some degree of personality disturbance is counterintuitive, but the evidence that the largest group, those with 'personality difficulty' covering two out of five of the population, differs significantly from those with no personality disturbance in the prevalence of a history of running away from home, police contacts, homelessness and sexual abuse, current employment status and primary and secondary care service use, shows that this separation is useful from both clinical and societal viewpoints. There are other challenges posed by the study that need to be answered.

\section{Greater societal dysfunction is to be expected in those with more personality disorder}

Even though the results might be regarded as expected they are to some extent counterintuitive. Thus, for example, it would not be expected that a person with both borderline and antisocial personality disorder (simple personality disorder) would have less dysfunction than someone with both histrionic and dependent personality disorder (complex personality disorder). Similarly, it appears obvious that severe personality disorder with a strong antisocial component would be associated with childhood delinquent behaviour, but for the 'severe' label more cluster pathology is needed and simple antisocial personality disorder does not appear to carry the same history or risks. In this context the finding that every single person in the severe personality disorder group had conduct disorder as a child (Table 3) is notable. In previous work, the possession of very severe pathology in one personality domain was found to be much less handicapping than milder pathology in more than one domain ${ }^{5}$ and this is confirmed in this study.

\section{The cluster system of personality classification is outmoded and inappropriate}

The cluster classification, despite many critics, is widely used in practice and does accord with the main domains of personality typology. ${ }^{21}$ In any revision of the classification of personality disorders it is unlikely that the main characteristics of the cluster system will be abandoned. This cannot be said for the individual categories of personality disorder. There has been great concern about the inadequacies of the current classification of these ever since the introduction of DSM-III, and its Axis II for personality disorder classification, 30 years ago. ${ }^{22}$ The operational criteria for the individual categories of personality disorder overlap greatly, ${ }^{23}$ some categories are so infrequently used they are virtually redundant ${ }^{24,25}$ yet personality disorder - not otherwise specified (NOS) is generally used more often than any other single diagnosis. ${ }^{26-28}$ One of the reasons for this is shown clearly in our study: when personality disturbance gets more severe the standard prototypes of disorder merge.

The severity model also allows dimensional and categorical approaches to personality disorder to be combined. A dimensional classification better reflects clinical reality ${ }^{24,25}$ but clinicians generally prefer a categorical system. ${ }^{29}$ By classifying personality pathology in terms of severity using a four- or five-level system the stigma associated with the diagnosis is reduced, the dimensional notion is retained in a clinically useful form, and, perhaps most importantly, the 'overflow' rippling across all personality prototypes at greater levels of severity is accommodated. Thus, in all cases those with the highest level of personality pathology - let us assume it would be named 'severe personality disorder' - would have at least two or three personality disorder prototypes associated with them and which would be included in the full diagnostic description. This would allow the severity/prototypical diagnoses to become much more homogeneous and ensure that all elements of personality pathology were included in any description of the severe groups. 

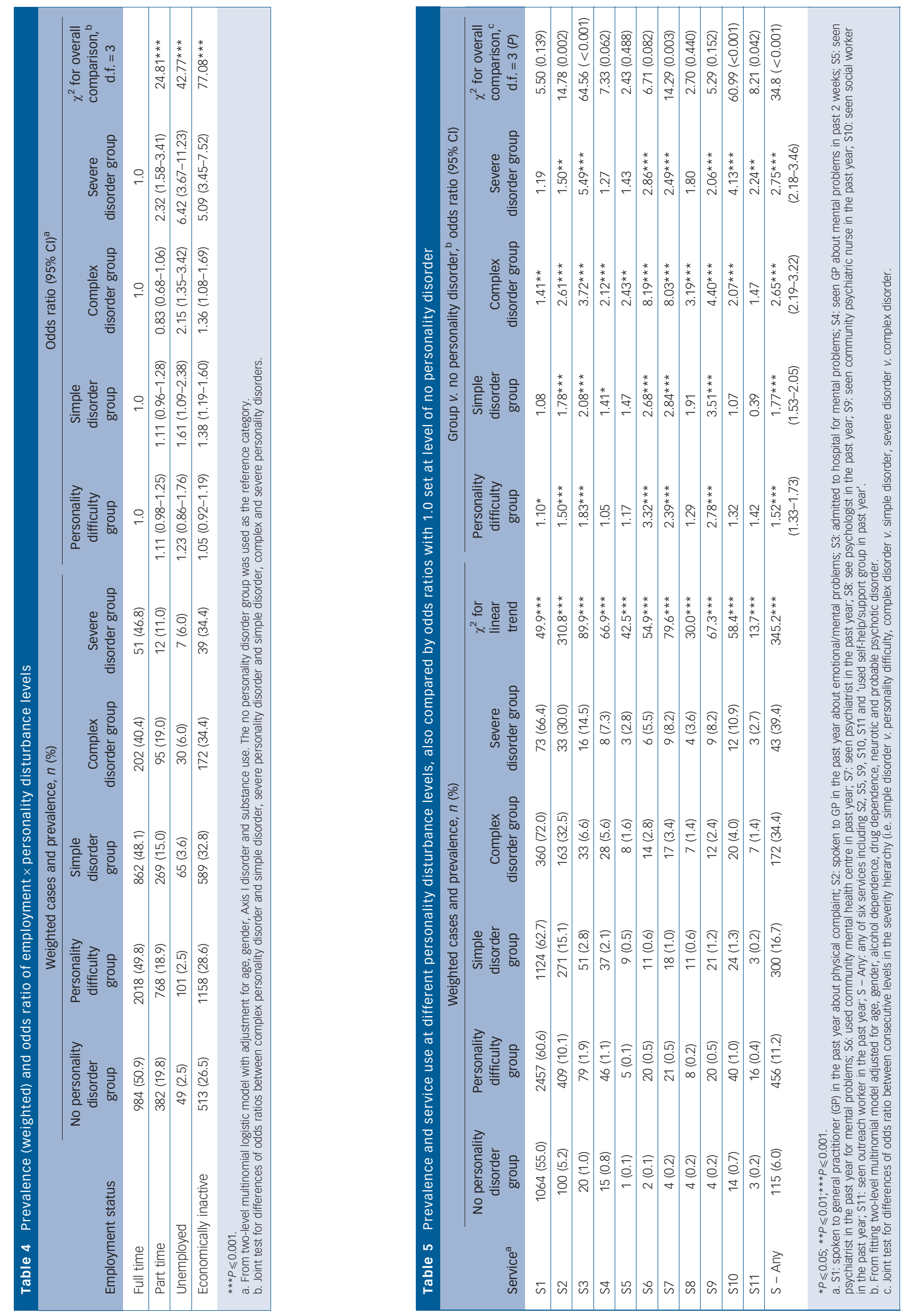


\begin{tabular}{|c|c|c|c|c|c|}
\hline & \multicolumn{5}{|c|}{ Odds ratio $(95 \% \mathrm{Cl})$} \\
\hline & $\begin{array}{l}\text { No personality } \\
\quad \text { disorder } \\
\text { (Reference group) }\end{array}$ & $\begin{array}{c}\text { Personality } \\
\text { difficulty group }\end{array}$ & $\begin{array}{c}\text { Simple } \\
\text { disorder group }\end{array}$ & $\begin{array}{l}\text { Complex } \\
\text { personality } \\
\text { disorder group }\end{array}$ & $\begin{array}{l}\text { Severe } \\
\text { personality } \\
\text { disorder group }\end{array}$ \\
\hline Probably psychosis & 1.0 & b & $3.00(1.77-5.10)$ & $6.69(3.86-11.57)$ & 12.68 (7.92-20.30) \\
\hline Neurotic disorder & 1.0 & $3.25(2.98-3.77)$ & 7.69 (6.70-8.82) & 23.57 (19.38-28.67) & 48.42 (35.39-66.26) \\
\hline Any drug dependence & 1.0 & $1.43(1.88-2.47)$ & $2.41(1.73-3.36)$ & $2.86(1.93-4.23)$ & 25.53 (18.30-35.63) \\
\hline Alcohol dependence ${ }^{c}$ & 1.0 & $1.52(0.25-1.85)$ & $2.36(1.90-2.93)$ & $3.94(3.11-4.98)$ & $6.62(5.13-8.54)$ \\
\hline \multicolumn{6}{|c|}{$\begin{array}{l}\text { a. From two-level multinomial logistic model taking into account geographic effects with adjustment for age, gender and employment. All four Axis I disorders were included } \\
\text { simultaneously in the same model. } \\
\text { b. No participants in this category. } \\
\text { c. Any alcohol dependence at a cut-off of the Severity of Alcohol Dependence Questionnaire }{ }^{20} \text { score at } 4 \text { or above. }\end{array}$} \\
\hline
\end{tabular}

In this way everyone could be placed on a spectrum with those with more persistent or recurring pathology being part of a personality diathesis, a possibly more accurate term than disorder ${ }^{30}$ and covering the spectrum of personality pathology more effectively. ${ }^{31}$

\section{Future classification of personality disorder}

We do not suggest that our method of recording severity is ideal. The algorithm derived in this study came from classification of data from a diagnostic system that has served its purpose and will be superseded, but even with this handicap, it offers a clinically useful tool that solves many of the puzzling questions being asked of those involved in making the ICD-11 and DSM-V revisions of personality disorder classification. This algorithm does not satisfy all the requirements of a revised classification; the definition of severity needs to have more than overlapping personality features in its description and the main lower-order groupings that form a more accurate definitional substrate of the current cluster system $^{32}$ need further testing. But the severity concept closely matches the concerns of clinicians and health planners, identifies the population that will need priority attention from our current resource-intensive treatments, ${ }^{33}$ and, by using graded levels of severity, helps to reduce stigma about a diagnosis that is reluctantly embraced by individuals with personality disorder, carers and clinicians.

The severity model also needs to decide whether or not to incorporate disturbance in social function into its definitions as the social handicap of personality disorder has been a core feature of the diagnosis since the time of Schneider. ${ }^{34}$ However, even in its current form ${ }^{5}$ it has already been shown to be a robust predictor of comorbid Axis I disorder in the 43093 people assessed in the National Epidemiologic Survey on Alcohol and Related Conditions (NESARC), ${ }^{35}$ in substantial trials of individuals assessed with repeat self-harm episodes, ${ }^{36,37}$ in a 12 -year follow up of 201 participants with persistent anxiety and depressive disorders $^{38}$ and, at the highest level of severity, in 75 people being assessed for severe personality disorder, in the new Dangerous and Severe Personality Disorder programme in England. ${ }^{39}$

One of the reasons why many policy makers, clinicians and planners of mental health services tend to ignore personality disorders is that the prevalence rates in the population of between 4 and $13 \%^{1-3}$ are so large as to be impossible to accommodate in service terms. The classification of this disorder using a system incorporating severity brings it down to a size and nature that makes it clinically relevant, manageable and worthy of closer consideration by mental health professionals of all persuasions.
Min Yang, MD, MPH, Division of Psychiatry, School of Community Health Science, University of Nottingham, Nottingham; Jeremy Coid, MD, FRCPsych, Queen Mary College, London, Forensic Psychiatry Research Unit, St Bartholomew's Hospital, London; Peter Tyrer, MD, Centre for Mental Health, Imperial College, London, UK

Correspondence: Professor Peter Tyrer, Centre for Mental Health, Imperial College, St Dunstan's Road, London W6 8RP, UK. Email: p.tyrer@imperial.ac.uk

First received 17 Feb 2010, final revision 18 May 2010, accepted 25 May 2010

\section{Funding}

This study was carried out by a grant from the UK Department of Health to the Office of National Statistics.

\section{References}

1 Torgersen S, Kringlen E, Cramer V. The prevalence of personality disorders in a community sample. Arch Gen Psychiatry 2001; 58: 590-6.

2 Huang $Y$, Kotov R, de Girolamo G, Preti A, Angermeyer M, Benjet $C$, et al DSM-IV personality disorders in the WHO World Mental Health Surveys. Br J Psychiatry 2009; 195: 46-53.

3 Coid J, Yang $M$, Tyrer $P$, Roberts A, Ullrich S. Prevalence and correlates of personality disorder in Great Britain. Br J Psychiatry 2006; 188: 423-31.

4 Blagov PS, Bradley R, Westen D. Under the axis II radar: clinically relevant personality constellations that escape DSM-IV diagnosis. J Nerv Ment Dis 2007; 195: 477-83.

5 Tyrer $\mathrm{P}$, Johnson $\mathrm{T}$. Establishing the severity of personality disorder. Am J Psychiatry 1996; 153: 1593-7.

6 Tyrer P. Challenges for the future. In Personality Disorders: Diagnosis, Management and Course (ed P Tyrer): 126-32. Arnold, 2000.

7 Crawford M, Koldobsky N, Tyrer P. Classifying personality disorder according to severity. J Person Disord 2010, in press.

8 Singleton N, Bumpstead R, O'Brien M, Lee A, Meltzer H. Psychiatric Morbidity among Adults Living in Private Households, 2000. TSO (The Stationery Office), 2001.

9 Kish L. Survey Sampling. John Wiley, 1965

10 Bebbington P, Nayani T. The Psychosis Screening Questionnaire. Int J Meth Psychiat Res 1994; 5: 11-9.

11 Lewis G, Pelosi AJ. Manual of the Revised Clinical Interview Schedule (CIS-R). Institute of Psychiatry, 1990.

12 Spitzer RL, Williams JBW, Gibbon M, First MB. The Structured Clinical Interview for DSM-II Axis II (SCID-II). American Psychiatric Press, 1990.

13 American Psychiatric Association. Diagnostic and Statistical Manual of Mental Disorder (4th edn) (DSM-IV). APA, 1994.

14 Reich J, Thompson WD. DSM-III personality disorder clusters in three populations. Br J Psychiatry 1987; 150: 471-5.

15 Singleton N, Meltzer H, Gatward R. Psychiatric Morbidity among Prisoners in England and Wales. TSO (The Stationery Office), 1998.

16 Ekselius L, Lindström E, von Knorring L, Bodlund O, Kullgren G. SCID I interviews and the SCID Screen questionnaire as diagnostic tools for personality disorders in DSM-III-R. Acta Psychiatr Scand 1994; 90: 120-3. 
17 Singleton N, Lee A, Meltzer H. Psychiatric Morbidity among Adults Living in Private Households, 2000: Technical Report: 19-26. Office for National Statistics, 2002.

18 Yang M. Multinomial regression. In Multilevel Modelling of Health Statistics (eds A Leyland \& H Goldstein): 107-26. John Wiley \& Sons, 2001.

19 Rasbash J, Browne W, Healy M, Cameron B, Charlton C. A User's Guide to MLwiN. Institute of Education, University of London, 2000.

20 Stockwell T, Murphy D, Hodgson R. The Severity of Alcohol Dependence questionnaire: its use, reliability and validity. Br J Addict 1983; 78: 145-55.

21 Tyrer $\mathrm{P}$, Mulder R, Crawford M, Newton-Howes G, Simonsen E, Ndetei $D$, et al. Personality disorder: a new global perspective. World Psychiatry 2010; 9: $56-60$.

22 American Psychiatric Association. Diagnostic and Statistical Manual of Mental Disorder (3rd edn) (DSM-IIII): 23. APA, 1980.

23 Livesley WJ, Jackson DN. Guidelines for developing, evaluating, and revising the classification of personality disorders. J Nerv Ment Dis 1992; 180: 609-18.

24 Widiger TA, Simonsen E. Alternative dimensional models of personality disorder. J Person Disord 2005; 19: 110-30.

25 Clark LA. Assessment and diagnosis of personality disorder: perennial issues and an emerging reconceptualization. Ann Rev Psychol 2007; 58: 227-57.

26 Verheul $\mathrm{R}$, Widiger TA. A meta-analysis of the prevalence and usage of the personality disorder not otherwise specified (PDNOS) diagnosis. J Person Disord 2004; 18: 309-19.

27 Verheul R, Bartak A, Widiger T. Prevalence and construct validity of Personality Disorder Not Otherwise Specified (PDNOS). J Person Disord 2007, 21: 359-70.

28 Wilberg T, Hummelen B, Pedersen G, Karterud S. A study of patients with personality disorder not otherwise specified. Compr Psychiatry 2008; 49: 460-8.

29 Spitzer RL, First MB, Shedler J, westen D, Skodol AE. Clinical utility of five dimensional systems for personality diagnosis: a "consumer preference" study. J Nerv Ment Dis 2008; 196: 356-74.
30 Tyrer P. Personality diatheses: a superior description than disorder. Psychol Med 2007; 37: 1521-5.

31 Mullins-Sweatt SN, Smit V, Verheul R, Oldham J, Widiger TA. Dimensions of personality: clinicians' perspectives. Can J Psychiatry 2009; 54: 247-55.

32 Widiger TA, Livesley WJ, Clark LA. An integrative dimensional classification of personality disorder. Psychol Assess 2009; 21: 243-55.

33 Chanen AM, Jackson HJ, McCutcheon LK, Jovev M, Dudgeon P, Yuen HP, et al. Early intervention for adolescents with borderline personality disorder using cognitive analytic therapy: randomised controlled trial. Br J Psychiatry 2008; 193: $477-84$.

34 Schneider K. Die psychopathischen Personlichkeiten [The Psychopathic Personalities]. Deutike, 1950.

35 Pulay AJ, Dawson DA, Ruan WJ, Pickering RP, Huang B, Chou P, et al. The relationship of impairment to personality disorder severity among individuals with specific axis I disorders: results from the national epidemiologic survey on alcohol and related conditions. J Person Disord 2008; 22: 405-17.

36 Tyrer $\mathrm{P}$, Tom B, Byford S, Schmidt U, Jones V, Davidson $\mathrm{K}$, et al. Differential effects of manual assisted cognitive behavior therapy in the treatment of recurrent deliberate self-harm and personality disturbance: the POPMACT study. J Personal Disord 2004; 18: 82-96.

37 Blasco-Fontecilla H, Baca-Garcia E, Dervic K, Peraz-Rodriguez MM, Saiz-Gonzalez MD, Saiz-Ruez J, et al. Severity of personality disorder and suicide attempt. Acta Psychiat Scand 2009; 119: 149-55.

38 Tyrer $\mathrm{P}$, Seivewright $\mathrm{H}$, Johnson $\mathrm{T}$. The Nottingham Study of Neurotic Disorder: predictors of 12 year outcome of dysthymic, panic and generalised anxiety disorder. Psychol Med 2004; 34: 1385-94.

39 Tyrer $\mathrm{P}$, Cooper S, Rutter D, Seivewright H, Duggan C, Maden T, et al. The assessment of dangerous and severe personality disorder: lessons from a randomised controlled trial linked to qualitative analysis. J Forens Psychiatr Psychol 2009; 20: 132-46. 